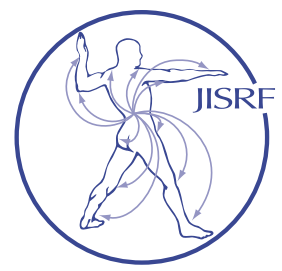

\title{
Impact of Anterior Cruciate Ligament Status on Early Satisfaction and Clinical Outcomes Following Total Knee Arthroplasty
}

\author{
Etemad-Rezaie, $A^{1}$; Edmiston, $T^{2}$; Kearns, $S^{2}$; Locker, $P^{2} ;$ Bohl, $D^{2}$; Sexton, $A^{2} ;$ Frank, $R^{3}$; Levine, $B^{2}$
}

\section{Abstact}

Background: While total knee arthroplasty (TKA) is a successful treatment for debilitating arthritis, up to $20 \%$ of patients may be dissatisfied with their outcome. One hypothesis for dissatisfaction is the distortion of native knee kinematics following sacrifice of the anterior cruciate ligament (ACL) during TKA. The purpose of this study was to determine the impact of ACL status at the time of surgery in patients undergoing Posterior Stabilized (PS) TKA for osteoarthritis (OA).

Methods: A consecutive prospective series of patients undergoing TKA by a single surgeon underwent prospective intraoperative assessment of their ACL status divided into three different groups:1) intact, 2) attenuated, or 3) deficient. Demographic, preoperative, intraoperative, and postoperative data were collected for each patient by two blinded, independent observers. Outcomes included patient satisfaction and Knee Society Score for Pain (KSS) and Function (KSF), Kellgren and Lawrence (K\&L), UCLA Activity Score (UCLA), Short Form-12 (SF12), EuroQol (EQ5D) and patient satisfaction.

Results: Of 116 patients, 33 (28.4\%) patients had an ACL deficient knee, 40 (34.5\%) patients had an attenuated ACL, and $43(37.1 \%)$ patients had an intact ACL. Those with absent ACL were significantly more likely to have a higher BMI $(\mathrm{p}=.007)$ and be male $(\mathrm{p}=.003)$. Patient with a deficient ACL had significantly lower preoperative KSF and higher $\mathrm{K} \& \mathrm{~L}$ scores $(\mathrm{p}=.009, \mathrm{p}=1.26 \times 10-7)$. Attenuated and deficient groups had the greatest change in SF-
12PCS scores at their one-year follow-up with increases of $9.9( \pm 10.0)$ and $10.8( \pm 8.0)$, respectively $(p=.037)$. No significant differences in overall postoperative KSS, KSF and satisfaction scores based on ACL status ( $\mathrm{p}=.574$ and $\mathrm{p}=.529$, respectively) were found.

Conclusion: In a relatively large series, patient with ACL deficiency were more likely to have worse pre-operative outcome scores and similar or better post-operative outcome scores. This suggests that those with ACL insufficiency may experience more subjective improvement from TKA. ACL status can be used as an additional surgical marker to help orthopaedic surgeons identify which patients would most benefit from TKA.

\section{Background}

Total knee arthroplasty (TKA) is considered a successful treatment for severe osteoarthritis $(\mathrm{OA})$ of the knee, often eliminating an affected patient's pain and discomfort [13]. However, as many as $20 \%$ of patients feel neutral, dissatisfied, or very dissatisfied about their TKA post-operatively [4]. This is evident with previously reported average post-operative Knee Society Function (KSF) scores ranging from $66.7-75.7[\underline{1}, \underline{2}, \underline{10}]$.

It is theorized that native knee structures such as the

Keywords: anterior cruciate ligament; ACL; total knee arthroplasty; TKA; clinical outcomes; satisfaction; impact Level of Evidence: III 
ACL may play an important role in patients achieving normal knee kinematics and satisfaction following their TKA. Patients undergoing TKA often exhibit variable histopathological changes in their ACL prior to surgery, which is thought to result in impaired knee joint stability and kinematics [11]. Multiple authors have reported degenerative changes being more severe with typically multiple compartment involvement of the knee with ACL deficiency $[\underline{6}, \underline{7}]$. As a result, it has been suggested that pre-operative ACL deficiency may precipitate degenerative patterns due to altered knee kinematics [16], and that lack of an ACL may lead to impaired knee kinematics for patients with end stage degenerative joint disease. Therefore, knowing the status of the ACL in the degenerative knee can be an important indication for TKA [11].

To the author's knowledge, no previous study has directly assessed ACL status and its impact on clinical outcome scores and patient satisfaction following TKA. Therefore, the purpose of this study is to determine the impact of a patient's pre-operative ACL status on post-operative outcomes following TKA. The hypothesis of this study is that a patient's prior ACL status has a significant impact in the pre-operative outcome measures. Specifically, the authors hypothesize that patients with absent or deficient ACLs will have worse pre-operative clinical scores, consistent with more advanced degenerative changes, and postoperatively, will have same or greater increase in their outcome measure scores. The results of this study will prove useful in understanding the role of natural knee kinematics in TKA and help to identify the role of ACL in degenerative joint disease of the knee. In addition, determining the prevalence of an intact ACL shed light on the possible utility of ACL-preserving knee arthroplasties for patients.

\section{Methods}

Following Institutional Review Board (15092303IRB01) approval, a consecutive series of 466 patients undergoing TKA by the senior investigator between September 1, 2013 and August 1, 2016 were queried using CPT code (27447). Patients under this code were considered for our prospective study. Inclusion criteria included patients over the age of 18 years who underwent Posterior Stabilized (PS) TKA and had their ACL status determined by the senior author intra- operatively. Exclusion criteria included patients that underwent a revision TKA, patients with a previous ACL reconstruction surgery, any patient with a prior open surgical procedure on the affected knee and any patient with missing or incomplete outcome scores at their one-year follow-up. A total of 116 patients out of
466 were deemed eligible. All patients undergoing TKA had their ACL status determined using a standard physical exam, as well as direct intra- operative visualization by a single surgeon. ACL statuses were categorized as: 1) intact, 2) present and weak in the case of fraying, partial tear or mild/moderate degenerative changes, or 3 ) deficient in the case of full thickness tear or severe degenerative changes. Preoperative, intraoperative, and postoperative data was collected from medical charts by blinded, independent observers. Data gathered included: ACL status, physical exam findings, age at the time of surgery, BMI, sex, medical comorbidities, surgical history, surgery laterality, and patient reported outcomes (PROs). PROs included Knee Society Score for Pain (KSS) and Function (KSF), UCLA Activity Score (UCLA), Short Form-12 (SF12), and EuroQol (EQ5D). Patient reported satisfaction was documented as: very satisfied (1), satisfied (2), satisfied with complaints (3), and unsatisfied (4).

\section{Statistics}

Statistical analyses were conducted using Stata version 13.1 (StataCorp, CollegeStation, TX). First, baseline and operative characteristics were compared between patients in the absent, attenuated, and intact ACL groups using Pearson's chi-squared test (for categorical outcomes) or ANOVA (for continuous outcomes). Second, clinical outcomes were compared between patients in the absent, attenuated, and intact ACL groups using ANOVA. These clinical comparisons were conducted for findings at the preoperative time point, for findings at the 1-year postoperative time point, and for the change between the preoperative and 1-year postoperative time point. All clinical comparisons were adjusted for age, sex, and BMI. The level of significance was set at $\mathrm{p}<0.05$.

\section{Results}

A total of 466 patients were enrolled, of which 116 met the inclusion criteria and had complete pre-operative and one-year follow-up data and represented the study population. Of these, 33 (28.4\%) had an ACL deficient knee, 40 (34.5\%) had an attenuated ACL, and $43(37.1 \%)$ had an intact ACL. ACL status was associated with male sex and higher BMI ( $\mathrm{p}=0.003$ and $\mathrm{p}=0.007$, respectively; Table 1).

Subsequent results are adjusted for age, sex, and BMI (Table 2). Patients with an absent or attenuated ACL were more likely to have a lower pre-operative KSF score than other patients $(\mathrm{p}=0.009)$. In addition, ACL absent group had a higher Kellgren and Lawrence $(\mathrm{K} \& \mathrm{~L})$ score $(\mathrm{P}=1.26 \times 10-7)$. ACL absent and attenuated groups expe- 
Table 1. Baseline and operative characteristics.*

\begin{tabular}{|c|c|c|c|c|}
\hline & Absent (33 patients) & Attenuated (40 patients) & Intact (43 patients) & P-value \\
\hline Age (years) & $67.6 \pm 10.1$ & $69.4 \pm 8.0$ & $65.5 \pm 8.5$ & 0.140 \\
\hline Male sex & $11(33.3 \%)$ & $6(15.0 \%)$ & $15(37.7 \%)$ & 0.003 \\
\hline
\end{tabular}

*Bolding indicates statistical significance.

Table 2. Clinical outcomes

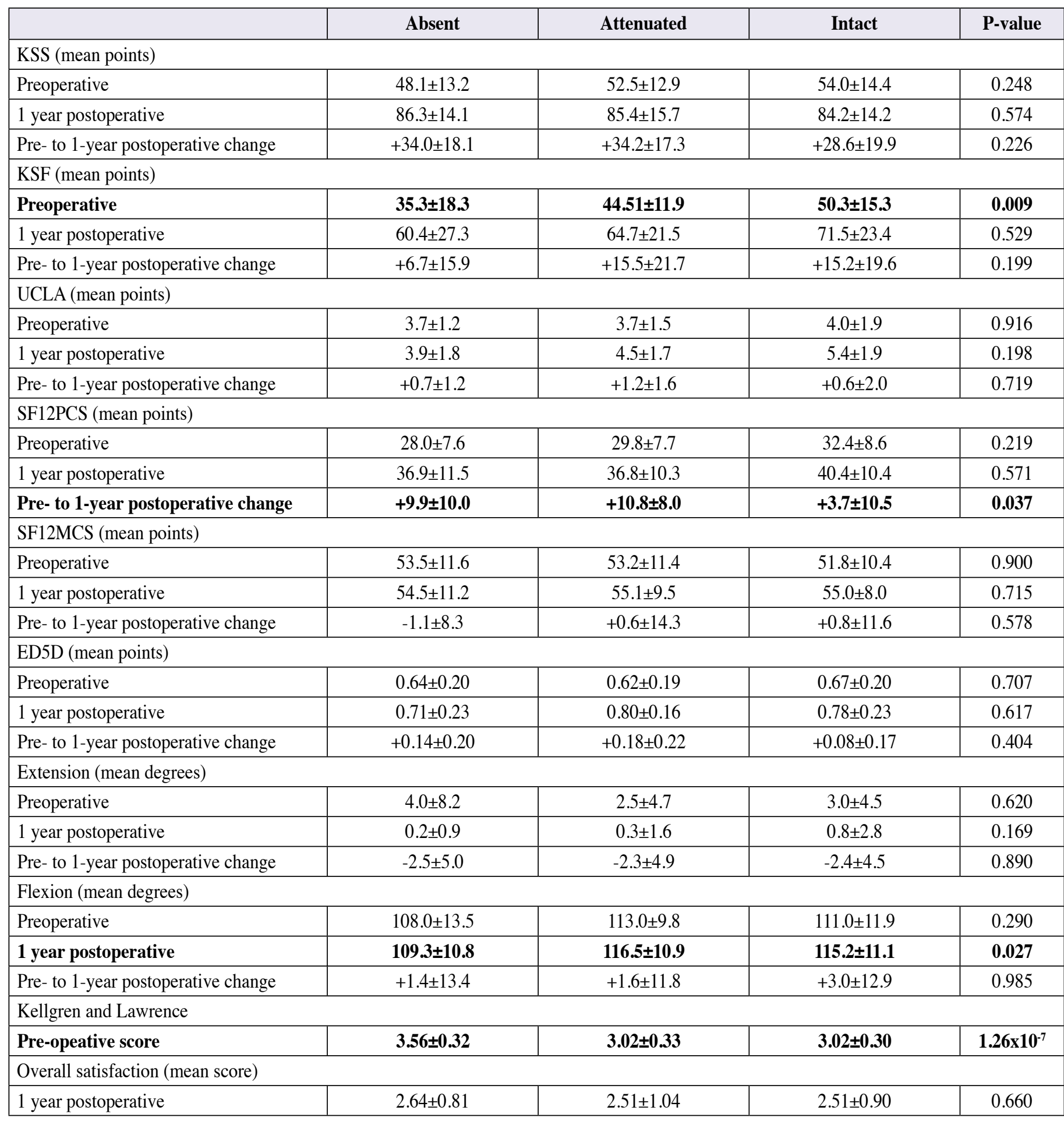

* Adjusted for age, sex, and body mass index. 
rienced greater improvement in SF12PCS from pre-operative to 1-year postoperative than patients with intact ACLs $(p=0.037)$. ACL- deficient patients had a decreased postoperative flexion at 1-year than other patients $(\mathrm{p}=0.027)$. However, the pre- to post-operative change in flexion did not show any difference across the three groups ( $>00.05$ ). All other clinical outcome assessments did not differ between the three groups. The mean satisfaction for all groups corresponded to satisfied, with no observed difference in patient satisfaction based on ACL status $(\mathrm{P}=0.660)$.

\section{Discussion}

Despite the success of TKA as a means for alleviating severe arthritis, a large number of patients remain dissatisfied with their outcomes. The role of patients' pre-operative ACL status has been hypothesized to affect both the post-operative outcome and satisfaction of patients. This study aimed to investigate whether patients with deficient ACLs had different post-operative outcomes compared to those with intact ACLs. We hypothesized that ACL pathology is associated with degenerative knee arthritis and patients with deficient ACLs experience similar or better outcomes at their 1-year follow-up.

The majority of the patients in this study (62.9\%) had an attenuated or deficient ACL, which is consistent with previously reported histologic studies. Cushner et al. and Mont et al. both demonstrated histologically that the ACL is part of the degeneration process that occurs in arthritic knees with abnormalities being present in $47 \%$ and $85 \%$ of knees undergoing TKA respectively $[\underline{5}, \underline{11}]$.

Our study results indicated that ACL-deficient knees had worse pre-operative KSF scores and a higher K\&L score, suggesting further clinical and radiological significance to the observed histological and radiographic findings in degenerative joint disease. Berend et al. demonstrated increased chondral and meniscal damage with more extensive osteophyte formation in ACL deficient knees [3]. In addition, Berend et al. discovered that ACL deficient knees were found to require more deformity correction and had lower pre-operative ROM [3]. These combined findings suggest that ACL deficiency is closely correlated with worsened degenerative joint disease both in terms of the patient's perspective as well as radiographic and clinical exam findings.

This study is one of the first studies that examined the role of ACL status in post-operative outcome measures of patients undergoing TKA. It was discovered that those with deficient ACL status exhibited similar or greater improvement across all their post-operative outcome measures up to one year. This can possibly be attributed to the abnormal knee kinematics that patients with deficient ACLs experience prior to their TKA, allowing for more subjective improvement post- operatively. Overall, ACL derangement can be used as one of the surgical markers of degenerative joint disease of the knee and can help orthopaedic surgeons identify potential candidates whom will benefit from a TKA.

Strengths of this study include 1) Visual inspection of ACL. The ACL of each patient was assessed intra-operatively by a single surgeon, 2) Blinded data collection. Observers collecting post-operative outcomes were blinded to the intra-operative ACL status of subjects, 3) Prospective nature of the study which allowed enough time for 1-year follow-up and inclusion of all the relevant outcome scores, and 4) Inclusion of multiple outcome scores. Nine total outcome scores were included in the study for a comprehensive assessment.

Limitations to this study include a relatively short, 1 year, follow-up period. Longer term follow- up may have revealed further changes in functional status following TKA between the three groups. However, 1 year is generally adequate to allow for a subsidence of residual pain and swelling caused by the surgery. Postoperatively, a 0-10 scale satisfaction survey may have yielded better estimation of patients' satisfaction compared to 1-4 scale that was used in this study. Lastly, having administrative measures placed to track patients and reduce the number of patients lost to follow-up would have minimized the possibility of attrition bias. However, 116 patients is still an adequate number in terms of sample size and overall power of the study.

\section{Conclusion}

In a relatively large series, over $60 \%$ of patients had a deficient or attenuated ACL at the time of TKA for OA. Compared to patients with an intact ACL, patients with ACL deficiency are more likely to have worse preoperative $\mathrm{KSF}$ and radiological K\&L scores, indicative of a more severe degenerative disease. However, those with ACL deficiency experience similar or possibly more relief from arthroplasty as demonstrated in SF12PCS outcome scores suggesting that abnormal knee kinematics prior to TKA may lead to more subjective improvement from arthroplasty compared to patients with intact ACLs prior to surgery. This indicates that natural knee kinematics and its alteration during surgery may play a role in clinical outcomes following TKA. As a result, ACL status can be used as an additional surgical marker to help orthopaedic surgeons 


\section{identify which patients would most benefit from TKA.}

\section{List of abbreviations}

TKA - Total Knee Arthroplasty

ACL - Anterior Cruciate Ligament

KSS - Knee Society Score

KSF - Knee Society Function

SF-12- Short Form 12

EQ5D - EuroQol

\section{References}

1. Argenson JN, Boisgard S, Parratte S, et al. Survival analysis of total knee arthroplasty at a minimum 10 years' follow-up: a multicenter French nationwide study including 846 cases. Orthop Traumatol Surg Res 2013;99(4):385.

2. Barrington JW, Sah A, Malchau H, et al. Contemporary cruciate-retaining total knee arthroplasty with a pegged tibial baseplate results at a minimum of ten years. J Bone Joint Surg Am 2009;91(4):874.

3. Berend, ME. ACL damage and deficiency is associated with more severe preoperative deformity, lower range of motion at the time of TKA. 2016; 12(3): 235-239.

4. Bourne RB, Chesworth BM, Davis AM, et al. Patient satisfaction after total knee arthroplasty: who is satisfied and who is not? Clin Orthop Relat Res 2010;468(1):57.

5. Cushner FD, La Rosa DF, Vigorita VJ, et al. A quantitative histologic comparison: ACL degeneration in the osteoarthritic knee. J Arthroplasty. 2003; 18(6): 687-92.

6. Hill CL, Seo GS, Gale D, et al. Cruciate ligament integrity in osteoarthritis of the knee. Arthritis Rheum. 2005; 52(3): 794-9.

7. Johnson AJ, Howell SM, Costa CR, et al. The ACL in the arthritic knee: how often is it present and can preoperative tests predict its presence? Clin Orthop Relat Res. 2013; 471(1): 181-8.

8. Komistek RD, Allain J, Anderson DT, Dennis DA, Goutallier D. In vivo kinematics for subjects with and without an anterior cruciate ligament. Clin Orthop Relat Res. 2002;404:315-325.

9. Lee GC, Cushner FD, Vigoritta V, et al. Evaluation of the anterior cruciate ligament integrity and degenerative arthritic patterns in patients undergoing total knee arthroplasty. J Arthroplasty. 2005; 20(1): 59-65.

10. Metsovitis SR, Ploumis AL, Chantzidis PT, et al. Rotaglide total knee arthroplasty: a long- term follow-up study. J Bone Joint Surg Am 2011;93(9):878.

11. Mont MA, Elmallah, RK, Cherian JJ, et al. Histopathological Evaluation of Anterior Cruciate Ligament in Patients Undergoing Primary Total Knee Arthroplasty. The Journal of Arthroplasty 2015pii: S0883-5403(15)00610-5

12. Mullaji AB, Marawar SV, Luthra M. Tibial articular cartilage wear in varus osteoarthritic knees: correlation with anterior cruciate ligament integrity and severity of deformity. J Arthroplasty. 2008; 23(1): 128-35.

13. NRJ. National Joint Registry. National Joint Registry for England and Wales: 11th Annual Report; 2017.

14. Sabouret P, Lavoie F, Cloutier JM. Total knee replacement with retention of both cruciate ligaments: a 22-year follow-up study. The Bone \& Joint Journal 2013;95$\mathrm{B}(7): 917-922$.

15. Schmidt R, Komistek RD, Blaha JD, Penenberg BL, Maloney WJ. Fluoroscopic analyses of cruciate-retaining and medial pivot knee implants. Clin Orthop Relat Res. 2003;410:139-147.

16. Stiehl JB, Komistek RD, Cloutier JM, Dennis DA. The cruciate ligaments in total knee arthroplasty: a kinematic analysis of 2 total knee arthroplasties. Journal of Arthroplasty 2000 Aug;15(5):545-550
SUBMISSION HISTORY

Submitted: April 27, 2020

Reviewed: May 29, 2020

Accepted: June 24, 2020

Published: July 20, 2020

\section{AUTHOR AFFILIATIONS}

1 Ali Etemad-Rezaie, MD, MS

Division of Orthopaedic Surgery University of Toronto, 149 College Street, 5th floor, Toronto, Ontario, Canada

2 Tori A. Edmiston, MD; Sean M. Kearns, MD; Philip H. Locker, MD; Daniel D. Bohl, MD, MPH; Andrew Sexton, BS; Brett Levine, MD, MS

Department of Orthopaedic Surgery, Rush University Medical Center, Chicago, Illinois, USA

3 Rachel M. Frank, MD

University of Colorado at Denver, Colorado, USA

(Direct inquires to Ali Etemad, ali.etemad@mail.utoronto.ca)

AUTHOR DISCLOSURES (combined, blinded statement)

- Royalties from a company or supplier (The following conflicts were disclosed) None

- Speakers bureau/paid presentations for a company or supplier (The following conflicts were disclosed) None

- Paid employee for a company or supplier (The following conflicts were disclosed) None

- Paid consultant for a company or supplier (The following conflicts were disclosed) Link, Merete, Exactech, DJO and Medacta

- Unpaid consultants for a company or supplier (The following conflicts were disclosed) Arthrex

- Stock or stock options in a company or supplier (The following conflicts were disclosed) None

- Research support from a company or supplier as a Principal Investigator (The following conflicts were disclosed) Zimmer-Biomet, Artelon

- Other financial or material support from a company or supplier (The following conflicts were disclosed) None

- Royalties, financial or material support from publishers (The following conflicts were disclosed) Slack INC, Human Kinetics, McGraw-Hill

- Medical/Orthopaedic publications editorial/governing board (The following conflicts were disclosed) Elseiver, Journal of Arthroplasty, Orthopedics

- Board member/committee appointments for a society (The following conflicts were disclosed) Orthopedics Today, Deputy Editor Arthroplasty Today, Patient Education and Research Committees AAHKS; AAOS Evaluation Committee

\section{COPYRIGHT \& OPEN ACCESS}

C) 2020 Etemad-Rezaie, Edmiston, Kearns, Locker, Bohl, Sexton, Frank, Levine. All rights reserved.

Authors retain copyright and grant the journal right of first publication with the work. Reconstructive Review is an open access publication and follows the Creative Commons Attribution-NonCommercial CC BY-NC. This license allows anyone to download works, build upon the material, OPEN 6 ACCESS and share them with others for non-commercial purposes as

Iong as they credit the senior author, Reconstructive Review, and the Joint Implant Surgery \& Research Foundation (JISRF). An example credit would be: "Courtesy of (senior author's name), Reconstructive Review, JISRF, Chagrin Falls, Ohio". 